\title{
Electrolyte-Gated Organic Field-Effect Transistor based-on a Solution Sheared Organic Semiconductor Blend
}

By Francesca Leonardi, Stefano Casalini, Qiaoming Zhang, Sergi Galindo, Diego Gutiérrez and Marta Mas-Torrent*

[*] Institut de Ciència de Materials de Barcelona (ICMAB-CSIC) and CIBER-BBN, Campus de la UAB, 08193, Bellaterra, Spain.

E-mail:mmas@icmab.es

Keywords: Solution Shearing, Electrolyte-Gated Organic Field-Effect Transistors, Printable Electronics, Organic Semiconductor Blend, Organic Large-Area Electronics.

Important advances have been achieved in printable electronics due to the discovery of high performing soluble organic semiconductors (OSCs) together with the development of a plethora of printing techniques. ${ }^{[1,2]}$ One successful approach to print organic semiconducting molecules consists of blending them with inert polymers in order to facilitate the material processing and to achieve higher device stability. ${ }^{[3-6]}$ Among the wide library of technological options, the exploitation of bar-assisted meniscus shearing (BAMS), along with soluble small molecule OSCs blended with an insulating polymer, represents a valuable route to achieve organic transistors featuring performance comparable to amorphous silicon ones. ${ }^{[4]}$ This is a non-contact technique that consists in pouring a blend solution in between a hot target substrate and a metallic bar forming a confined meniscus. As the bar is withdrawn, a fast crystallization of the film takes place at the substrate-solution-air interfaces, forming a nanometer-thick film. Furthermore, BAMS is a low cost and high throughput technique compatible to roll-to-toll processes (Fig.1a). ${ }^{[4]}$ It is widely accepted that one of the most promising applications of organic transistors is to employ them in sensing devices. ${ }^{[7-9]}$ In this direction, electrolyte-gated organic field-effect transistors (EGOFETs) are recently gaining much attention because they can operate in water (Fig.1b), and thus, can be directly turned into biochemical applications. ${ }^{[10-14]}$ However, to our knowledge, all reported EGOFET 
devices have been fabricated by spin coating or thermal evaporation of the OSC. ${ }^{[15]}$ Here, we report the fabrication of an EGOFET employing the BAMS technique and as active material a blend of dibenzo-tetrathiafulvalene (DB-TTF) with polystyrene (PS), as shown in Fig.1c. We demonstrate that EGOFETs can be successfully fabricated by printing techniques compatible with upscaling and, remarkably, that the use of semiconducting blends provides high performing and reproducible devices with enhanced stability in aqueous environment.

The EGOFET architecture takes advantage of the aqueous solution as gate dielectric (Fig.1b). The transversal electrical field due to the source-gate voltage $\left(V_{G S}\right)$ yields the formation of two electrical double layers (EDLs): one placed at the gate-electrolyte interface and the other one at the OSC-electrolyte interface. These EDLs can reach tens of $\mu \mathrm{F} / \mathrm{cm}^{2}$, hence EGOFETs are capable to work at operational voltages lower than $1 \mathrm{~V}$ featuring high charge carriers densities $\left(Q^{\prime}\right) .{ }^{[11,16]}$ Films of DB-TTF:PS in a ratio 1:2 were fabricated as published elsewhere. ${ }^{[4]}$ Optical microscopy allows us to observe large crystalline domains with grain size of $200 \times 200 \mu \mathrm{m}^{2}$ (Fig.S1). BAMS does not enable to reach the thermodynamic equilibrium during the deposition process; ${ }^{[17]}$ as a result the above-mentioned domains are distinctive of the DB-TTF $\gamma$-phase, which is the kinetically favoured one. ${ }^{[18]}$ The morphology of the active material is another key factor for thin-film transistors. ${ }^{[19]}$ The DB-TTF:PS thin film shows an excellent homogeneity onto both Au electrodes and $\mathrm{SiO}_{\mathrm{x}}$ channel featuring a root mean square roughness constantly lower than $10 \mathrm{~nm}$ (Fig.S2). Furthermore, Atomic Force Microscopy (AFM) and Energy Filtered Transmission Electron Microscopy (EF-TEM) have been used in order to define the total thickness of our thin-film that is in the range of $30 \div 35 \mathrm{~nm}$. In particular, EF-TEM provided the images related to the vertical cross-section of the channel device (see experimental section for further information), where the coated organic film is sandwiched between $\mathrm{SiO}_{\mathrm{x}}$ and Pt layers (Fig.1c). This image is not only consistent with the AFM results (Fig.S3), but also points out the clear vertical phase separation between DB-TTF and PS. ${ }^{[4,20]}$ The ca. $10 \mathrm{~nm}$ thick DB-TTF layer is sitting on top 
of the PS one, which is in direct contact with $\mathrm{SiO}_{\mathrm{x}}$. Although not resolvable by means of EFTEM, it was previously described that DB-TTF crystals are also covered by an ultra-thin PS capping layer providing device stability to the outer environment. ${ }^{[4 a]}$

Prior to the electrolyte-gating measurements, we have systematically characterized the electrical performance of DB-TTF:PS coating by using the bottom-gate configuration, since our substrate of choice is $\mathrm{Si} / \mathrm{SiO}_{\mathrm{x}}$, yielding devices with mobility values of around $0.2 \mathrm{~cm}^{2} / \mathrm{Vs}$ (Fig.S4). This electrical cross-check along with the optical one allowed us to verify systematically the quality of our blend coating. Afterwards, the electrical performance of [DB-TTF:PS]-based EGOFETs have been measured in bi-distilled water which is the aqueous benchmark media. It was found that the pure DB-TTF coating shows a poor electrical modulation together with a marked instability within few $V_{G S}$ cycles (Fig.S5). In sharp contrast, the [DB-TTF:PS]-based EGOFET showed an outstanding performance. These devices were electrically characterized by acquiring I-V transfer (Fig.2a) and output (Fig.2b) characteristics in a $V_{G S}$ range between $+950 \mathrm{mV}$ and $+350 \mathrm{mV}$. As shown in Fig.2a, leakage current as low as tens of $\mathrm{nA}$ and no hysteresis are two typical fingerprints of the electrostatic coupling between gate electrode and the active coating. ${ }^{[21]}$ Due to the fact that the ON/OFF transition is occurring at positive $V_{G S}$ values, we have performed impedance spectroscopy in the same potential window in order to extract the capacitance that governs the electrostatic coupling between gate electrode and DB-TTF:PS thin-film. ${ }^{[13]}$ These measurements exploit the same EGOFET layout by short-circuiting Source/Drain (S/D) electrodes, which act as counter electrode (see experimental section). Moving from $+950 \mathrm{mV}$ to $+350 \mathrm{mV}$, the capacitance undergoes a 4-fold increase due to charge carriers accumulation within the DBTTF:PS film, reaching values as high as $1.2 \mu \mathrm{F} / \mathrm{cm}^{2}$ (Fig.2c). This value is coherent with other devices featuring OSC thin-films such as poly(3-hexylthiophene-2,5-diyl) (P3HT) or pentacene. ${ }^{[22,23]}$ Furthermore, the direct comparison with a reference substrate (namely uncoated gold electrode) does clearly demonstrate that the DB-TTF:PS thin-film is 
responsible for the electrical modulation observed in the I-V characteristics (Fig.2c). According to the standard model describing OFET performance, we have extracted both $\mu$ and $V_{t h}$ by using the following equation:

$$
I_{D S}=\frac{W}{2 L} C_{i} \mu\left(V_{G S}-V_{t h}\right)^{2}
$$

where $W$ and $L$ are the channel width and length, $C_{i}$ is the capacitance per unit area of the gate insulator, $\mu$ is the charge-carrier mobility and $V_{t h}$ is the threshold voltage. Furthermore, the electronic transconductance, $g_{m}$, along with the charge carriers density, $Q^{\prime}$, have been further calculated by differentiating the I-V transfer characteristics and by integrating the capacitance trend as a function of $V_{G S}$, respectively (Fig.S6). ${ }^{[13]}$ All the values are listed in Table 1 . As well-known in literature, the exploitation of high- $k$ dielectrics leads to an intrinsic lowering of the charge carriers mobility due to the stronger influence of interface defects and surface roughness. ${ }^{[19,22]}$ Although we notice the same behaviour, this effect has not been so pronounced here as it is typically observed in other EGOFET devices. ${ }^{[24]}$ In fact the total decrease of the field-effect mobility is less than 1 order of magnitude moving from airoperated to liquid-gated devices. Although this issue is beyond the scope of this work, the moderate loss of mobility is likely ascribable to the presence of an ultra-thin capping layer of polystyrene atop of DB-TTF layer and the high homogeneity and crystallinity of the films.

Since EGOFETs belong to the family of the organic transistors, they have a wide range of applications due to their excellent sensitivity intrinsically intertwined with their capability of amplification. ${ }^{[7-9,11,12]}$ Among these applications, EGOFETs can be operated as electronic transducers for tracking biological events. ${ }^{[25]}$ According to the nature of the external stimuli to be monitored, switching speeds $(\tau)$ along with different potentiometric sensitivities are demanded. ${ }^{[26]}$ For this reason, these two figure of merits have been characterized by applying square gate-source pulses $\left(\Delta V_{G S}\right)$ during the EGOFET operation: the former parameter, namely $\tau$, was extracted by an amplitude pulse equal to $350 \mathrm{mV}$ with an integration 
measurement time of $200 \mu \mathrm{s}$; the latter was defined by applying a series of pulses featuring different amplitudes (e.g. from $100 \mathrm{mV}$ up to $100 \mu \mathrm{V}$ ) with a integration measurement time of 20 ms. The charging and discharging processes have been exponentially fitted (viz. $\left.\Delta I_{D S} \propto e^{ \pm(t / \tau)}\right)$, and they result to be symmetric featuring a $\tau$ equal to $4 \mathrm{~ms}$ and $7 \mathrm{~ms}$, respectively (Fig. 3a). On the other hand, the lowest limit of detection of potentiometric sensitivity is set at $100 \mu \mathrm{V}$ featuring a signal to noise ratio equal to 50 (Fig. 3b). These values are aligned to the state-of-the-art values reported for EGOFETs.

As previously stated, our EGOFETs show an excellent stability, as a result we decided to perform three type of electrical characterization: i) bias-stress measurements, ${ }^{[27]}$ ii) shelfstability ${ }^{[4]}$ and iii) in-situ real-time monitoring. ${ }^{[28]}$ These measurements focus on three distinct form of electrical stresses. The former one, "bias-stress", is a well-known phenomenon affecting the air-operated organic transistors. ${ }^{[27]}$ In our case, we applied $V_{G S}=+300 \mathrm{mV}$ and $V_{D S}=-50 \mathrm{mV}$ for $1 \mathrm{~min}$, and then we collected the I-V transfer characteristic in saturation regime. These devices clearly show a high-grade of stability within an hour of continuous stress (Fig.4a). A moderate increase of the OFF current together with a positive threshold voltage shift are hinting at a partial diffusion of ions within the DB-TTF:PS film (viz. electrochemical doping). The second electrical test (viz. shelf-life) is concerning single-spot measurements within a certain period of time. For this reason, we monitored the devices once per day for one week by acquiring I-V transfer characteristic, and afterwards the device was rinsed, dried and stored. As shown in Fig. 4b, our device can operate after one week, and it undergoes only a 35\% of current loss. The last stressing test, namely in-situ real-time monitoring, deals with the concrete possibility to track continuously an aqueous media, where is taking place an event of interest, such as an enzymatic reaction, a host-guest recognition, a neuronal spiking etc. This test consists of applying a $V_{G S}=+350 \mathrm{mV}$ and $V_{D S}=-400 \mathrm{mV}$ (namely saturation regime as operation mode) for several hours and acquiring $I_{D S}$. The recorded current reaches a plateau within few hours and it does not show any occurrence of 
electrical worsening (Fig.4c). As previously mentioned, bi-distilled water was selected as benchmark in order to test the electrical features of DB-TTF:PS coating. However, this aqueous media is rather far from real matrixes. For this reason, we verified the electrical performances of our electronic devices operated in aqueous solution of $\mathrm{NaCl}$ with a concentration of $100 \mathrm{mM}$. I-V transfer and output characteristics show clearly how [DBTTF:PS]-based EGOFETs can operate in this stressful environment (see Fig.S7a and Fig.S7b). The major effect is a negative $V_{t h}$ shift likely ascribable to an electrostatic screening towards polar dopant agents close to the conductive channel, as already verified for other similar devices. ${ }^{[29]}$ As reported in Fig.S7c, the capacitance of our system maintains the same ON/OFF modulation with respect to $V_{G S}$, but its values are higher due to the abrupt increase of the ionic strength. Concerning the potentiometric sensitivity no effects are observed in terms of absolute value (viz. $100 \mu \mathrm{V}$ ), however the signal/noise ratio is reduced by a factor of 5 (see Fig. S8). Since our devices show an excellent stability, we tested electrically in an even harsher environment, like $\mathrm{NaCl} 1 \mathrm{M}$ (Fig.S9). No failure has been observed by stressing the devices in the same experimental conditions. Although the electrical worsening is faster in the bias stress measurements, the shelf-stability is unaffected with respect to bi-distilled water. The real-time monitoring shows an additional depolarization process within the first 90 minutes, nonetheless it was possible to measure up to 9 hours without particular problems. Considering all above, these experiments prove that the here-reported EGOFETs can be benchmarked in real samples whose ionic strength is comparable to our reference solutions. In conclusion, we have successfully fabricated an electrolyte-gated organic field-effect transistor based on a DB-TTF:PS blend and deposited by BAMS, a low-cost technique compatible with roll-to-roll processes. The electrical performances are aligned to the state-ofthe-art in this field, where other OSCs like pentacene, ${ }^{[30]} \mathrm{P} \mathrm{HT}^{[11]}$ and $\alpha$-sexithiophene ${ }^{[31]}$ have been successfully used. Even though this device has not been further sophisticated in terms of layout, it shows promising features as follows: i) operational frequency $\sim 200 \mathrm{~Hz}$, ii) 
potentiometric sensitivity down to $100 \mu \mathrm{V}$ and iii) an excellent electrical stability promoted by the PS blending. For this reason, these devices can be considered promising candidates for both off-line and in-situ real-monitoring tests according to the purpose demanded.

\section{Experimental}

Materials: Dibenzo-tetrathiafulvalene, Polystyrene $\left(\mathrm{M}_{\mathrm{w}} \sim 3000 \mathrm{~g} \mathrm{~mol}^{-1}\right)$ and Chlorobenzene (CB) were purchased from Sigma-Aldrich and used without further purification. $0.5 \mathrm{~mm}$ diameter platinum wire was purchased from Sigma-Aldrich and solvent cleaned before each use. Polydimethilsiloxane (Sylgard ${ }^{\circledR} 184$ silicone elastomer kit) was purchased from Dow Corning Corporation.

\section{Experimental Setup:}

Chlorobenzene solutions (4 wt \%) of DB-TTF/PS were prepared at a volume ratio of 1:2 DBTTF:PS. Thin-films of organic blends were deposited at ambient conditions using a homemade bar coater at speed around $1 \mathrm{~cm} \mathrm{~s}^{-1}$ and keeping the substrates at $105{ }^{\circ} \mathrm{C}$. Our devices were fabricated on heavily doped n-type Si wafer (purchased from Si-mat) with a $200 \mathrm{~nm}$ thick layer of $\mathrm{SiO}_{\mathrm{x}}$. Before solution coating, source (S) and drain (D) electrodes were patterned using photolithography and a $\mathrm{Cr} / \mathrm{Au}(5 \mathrm{~nm} / 40 \mathrm{~nm})$ layer was deposited though thermal evaporation (system Auto 306 from Boc Edwards). The W/L ratio was fixed to 690 (i.e. $20700 \mu \mathrm{m}$ and $30 \mu \mathrm{m}$ as $W$ and $L$, respectively). Electrical measurements were acquired at ambient conditions using an Agilent 5100A and Easy Expert software connected to the samples with a SÜSS probe station. Top gate platinum wire was immersed in a droplet of electrolyte confined on the device area by means of a poly(dimethylsiloxane) pool. I-V transfer characteristics have been performed with scan rate equal to $60 \mathrm{mV} / \mathrm{s}$.

Optical microscope pictures were taken using an Olympus BX51 equipped with polarizer and analyzer. Surface topography was examined by a 5500LS SPM system from Agilent Technologies and subsequent data analysis was performed by using Gwyddion 2.41 software. 
Energy filtered transmission electron microscopy (EF-TEM) measurements were performed using a JEOL 2010-FEG (200 kV) microscope equipped with a Gatan Imaging Filter for EFTEM imaging mode. EF-TEM images were acquired with a slit width of $3 \mathrm{eV}$ centered at 23 eV (silicon oxide plasmon peak).

EF-TEM samples were prepared by using focused ion beam (Neon 40, Carl Zeiss) equipped with a micromanipulator (MM3A, Kleindiek). First, e-beam and ion beam assisted Pt deposition was performed to create a protective Pt layer on the region of interest in order to prevent Ga ion damage coming from ion beam milling. Then, the bulk material on both sides of the protective layer was removed by milling holes with ion currents of $10 \mathrm{nA}, 2 \mathrm{nA}$ and $500 \mathrm{pA}$ at $30 \mathrm{kV}$ until a lamella with dimensions of about $10 \mu \mathrm{m}$ length and $1 \mu \mathrm{m}$ width was created. Using the micromanipulator the lamella was then lifted out, transferred and welded to the TEM grid by Pt deposition. To achieve electron transparency, a small window $4 x 3 \mu \mathrm{m}$ in the lamella was thinned to about $60 \mathrm{~nm}$ by subsequent polishing steps with ion currents of 50 and $10 \mathrm{pA}$ at $30 \mathrm{kV}$. In order to reduce the ion beam induced amorphization, a final polishing step was performed with an ion current of $20 \mathrm{pA}$ at $5 \mathrm{kV}$.

Electrochemical Impedance Spectroscopy (EIS) was performed using a Novocontrol AlphaAN impedance analyser equipped with POT/GAL 30V/2A electrochemical interface.

EIS experiments have been directly carried on EGOFETs in a two electrodes configuration: Pt wire as working electrode and S/D as counter electrode. All the electrochemical data are normalized by the following areas: $0.0062 \mathrm{~cm}^{2}$ (viz. channel), $0.019 \mathrm{~cm}^{2}$ (viz. channel together with interdigitated electrodes). EIS response was recorded in MilliQ $\mathrm{H}_{2} \mathrm{O}$ and $\mathrm{NaCl} 100 \mathrm{mM}$ at seven different $\mathrm{DC}$ voltages, starting from $+900 \mathrm{mV}$ to $+300 \mathrm{mV}$ with constant $\mathrm{AC}$ amplitude of $10 \mathrm{mV}$ spanning from $10^{6} \mathrm{~Hz}$ to $10^{-1} \mathrm{~Hz}$.

\section{Acknowledgements}

The authors thank the ERC StG 2012-306826 e-GAMES project, the Networking Research Center on Bioengineering, Biomaterials and Nanomedicine (CIBER-BBN), the DGI (Spain) project BE-WELL CTQ2013-40480-R, the Generalitat de Catalunya (2014-SGR-17) and the 
Spanish Ministry of Economy and Competitiveness, through the 'Severo Ochoa' Programme for Centres of Excellence in R\&D (SEV- 2015-0496). F.L. gratefully acknowledges the "Juan de la Cierva" programme. The research leading to these results has received funding from the People Programme (Marie Curie Actions) of the Seventh Framework Programme of the European Union (FP7/2007-2013) under REA grant agreement no.600388 (TECNIOSpring programme), and from the Agency for Business Competitiveness of the Government of Catalonia, ACCIÓ. Q. Z. acknowledges the China Scholarship Council, the National Natural Science Foundation (NSF) of China (11404266) and the Fundamental Research Funds for the Central Universities (Grant No. XDJK2011C041). Q. Z. is enrolled in the Materials Science PhD Program of Universitat Autònoma de Barcelona. The authors gratefully acknowledge Prof. J. Puigdollers for the useful discussions ((Supporting Information is available online from Wiley InterScience or from the author)).

Received: ((will be filled in by the editorial staff))

Revised: ((will be filled in by the editorial staff)) Published online: ((will be filled in by the editorial staff))

[1] G. Giri, E. Verploegen, S.C.B. Mannsfeld, S. Atahan-Evrenk, D.H. Kim, S. Y. Lee, H.A. Becerril, A. Aspuru-Guzik, M. F. Toney and Z. Bao, Nature 2011, 480, 504-509.

[2] Y. Xu, C. Liu, D. Khim, Y.-Y. Noh, Phys. Chem. Chem. Phys. 2015, 17, 26553-26574.

[3] M. R. Niazi, R. Li, E. Qiang Li, A. R. Kirmani, M. Abdelsamie, Q. Wang, W. Pan, M. M. Payne, J. E. Anthony, D.-M. Smilgies, S. T. Thoroddsen, E. P. Giannelis, A. Amassian, Nat. Commun. 2015, 6, 8598.

[4] a) F. G. del Pozo, S. Fabiano, R. Pfattner, S. Georgakopoulos, S. Galindo, X. Liu, S. Braun, M. Fahlman, J. Veciana, C. Rovira, X. Crispin, M. Berggren, M. MasTorrent, Adv. Funct. Mater. 2016, 26, 2379-2386. b) I. Temiño, F. G. Del Pozo, M. R. Ajayakumar, S. Galindo, J. Puigdollers and M. Mas-Torrent, Adv. Mater. Technol., 2016, doi:10.1002/admt.201600090. c) S. Georgakopoulos, F. G. del Pozo, M. Mas-Torrent, J. Mater. Chem. C 2015, 3, 12199-12202.

[5] R. Hamilton, J. Smith, S. Ogier, M. Heeney, J. E. Anthony, I. McCulloch, J. Veres, D. D. C. Bradley, T. D. Anthopoulos Adv. Mat. 2009, 21, 1166-1171.

[6] L. Kergoat, N. Battaglini, L. Miozzo, B. Pirot, M-C Pham, A. Yassar and G. Horowitz, Org. Electron. 2011, 12, 1253-1257.

[7] S. Casalini, F. Leonardi, T. Cramer, F. Biscarini, Org. Electron. 2013, 14, 156-163. 
[8] M. Y. Mulla, E. Tuccori, M. Magliulo, G. Lattanzi, G. Palazzo, K. Persaud and L. Torsi Nat. Comm. 2015, 6, 6010.

[9] S. Casalini, A. C. Dumitru, F. Leonardi, C. A. Bortolotti, E. T. Herruzzo, A. Campana, R. F. De Oliveira, T. Cramer, R. Garcia and S. Casalini ACS Nano, 2015, 9, 5051-5062.

[10] M. J. Panzer, C. D. Frisbie, Adv. Mater. 2008, 20, 3177-3180.

[11] L. Kergoat, B. Piro, M. Berggren, G. Horowitz, M.-C. Pham, Anal. Bioanal. Chem. 2012, 402, 1813-1826.

[12] G. Foschi, F. Leonardi, A. Scala, F. Biscarini, A. Kovtun, A. Liscio, A. Mazzaglia and S. Casalini, Nanoscale, 2015, 7, 20025-20032.

[13] H. Kim, K. Hong, W. Xie, K. H. Lee, S. Zhang, T. P. Lodge, C. D. Frisbie, Adv. Mater. 2013, 25, 1822-1846.

[14] T. Cramer, A. Campana, F. Leonardi, S. Casalini, A. Kyndiah, M. Murgia and F. Biscarini, J. Mat. Chem. B, 2013, 1, 3728-3741.

[15] D. Wang, V. Noël and B. Piro, Electronics, 2016, 5, 9.

[16] L. Herlogsson, X. Crispin, N. D. Robinson, M. Sandberg, O.-J. Hagel, G. Gustafsson, M. Berggren, Adv. Mater. 2007, 19, 97-101.

[17] S. Fabiano, B. Pignataro, Chem. Soc. Rev. 2012, 41, 6859-6853.

[18] A. Brillante, I. Bilotti, R. G. Della Valle, E. Venuti, S. Milita, C. Dionigi, F. Borgatti, A. N. Lazar, F. Biscarini, M. Mas-Torrent, N. S. Oxtoby, N. Crivillers, J. Veciana, C. Rovira, M. Leufgen, G. Schmidt, L. W. Molenkamp, CrystEngComm 2008, 10, 18991909.

[19] K. Asadi, Y. Wu, F. Gholamrezaie, P. Rudolf, P. W. M. Blom, Adv. Mater. 2009, 21, 4109-4114.

[20] M. R. Niazi, R. Li, M. Abdelsamie, K. Zhao, D. H. Anjum, M. M. Payne, J. Anthony, D.-M. Smilgies, A. Amassian, Adv. Funct. Mater. 2015, 26, 2371-2378.

[21] L. Kergoat, B. Piro, M. Berggren, M.-C. Pham, A. Yassar, G. Horowitz, Org. Electron. 
2012, 13, 1-6.

[22] L. Kergoat, L. Herlogsson, D. Braga, B. Piro, M-C. Pham, X. Crispin, M. Berggren and G. Horowitz, Adv. Mater., 2010, 22, 2565-2569.

[23] T. Cramer, A. Kyndiah, M. Murgia, F. Leonardi, S. Casalini and F. Biscarini, Appl. Phys. Lett., 2012, 100, 143302.

[24] O. Knopfmacher, M. L. Hammock, A. L. Appleton, G. Schwartz, J. Mei, T. Lei, J. Pei and Z. Bao, Nat. Comm., 2014, 5, 2954.

[25] T. Cramer, B. Chelli, M. Murgia, M. Barbalinardo, E. Bystrenova D. M. De Leeuw and F. Biscarini, Phys. Chem. Chem. Phys., 2013, 15, 3897-3905.

[26] D. Khodagholy, M. Gurfinkel, E. Stavrinidou, P. Leleux, T. Herve, S. Sanaur, G. G. Malliaras, Appl. Phys. Lett. 2011, 99, 163304.

[27] P. A. Bobbert, A. Sharma, S. G. J. Mathijssen, M. Kemerink, D. M. de Leeuw, Adv. Mater. 2012, 24, 1146-1158.

[28] A. Campana, T. Cramer, D. T. Simon, M. Berggren, F. Biscarini, Adv. Mater. 2014, 26, 3874-3878.

[29] F. Chen, J. Xia, N. Tao, Nano Lett. 2009, 9, 1621-1625.

[30] G. Foschi, F. Leonardi, A. Scala, F. Biscarini, A. Kovtun, A. Liscio, A. Mazzaglia, S. Casalini, Nanoscale 2015, 7, 20025-20032.

[31] F. Buth, A. Donner, M. Sachsenhauser, M. Stutzmann, J. a Garrido, Adv. Mater. 2012, 24, 4511-4517. 


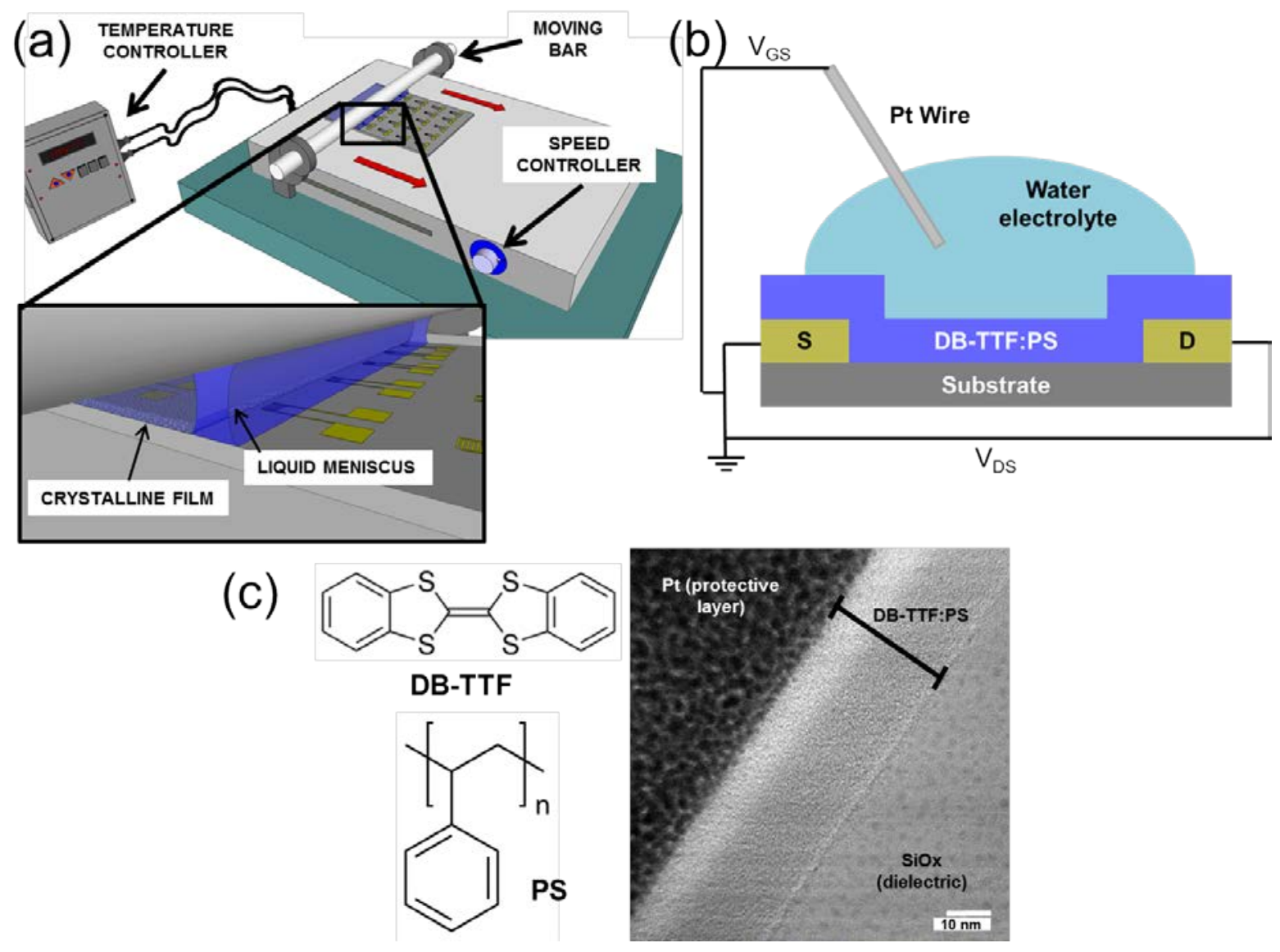

Figure 1. (a) Representative sketch of the Bar-Assisted Meniscus Shearing (BAMS) method. The inset depicts the liquid meniscus under the cylindrical bar and the crystalline film formation. (b) Vertical cross-section of our EGOFET. (c) Left. Chemical formulas of DB-TTF and PS. Right. EF-TEM image of the DB-TTF:PS coating. 


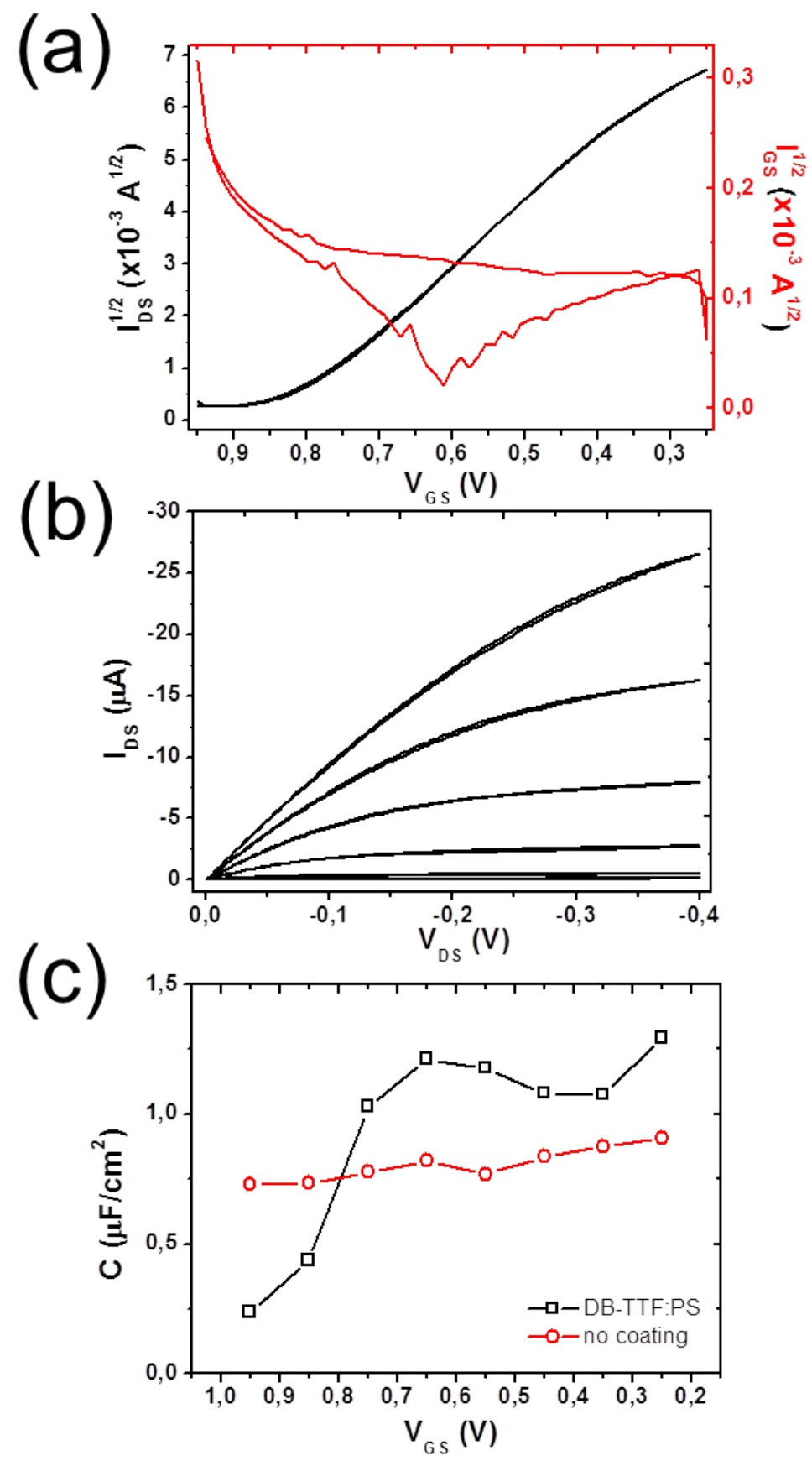

Figure 2. (a) I-V transfer characteristic of DB-TTF:PS EGOFET $\left(V_{D S}=-0.4 \mathrm{~V}\right)$; (b) I-V output characteristic $\left(V_{G S}=0.9 \mathrm{~V} \div 0.3 \mathrm{~V}\right.$ with step of $0.1 \mathrm{~V}$ ); (c) Capacitance versus $V_{G S}$ plot of [DB-TTF:PS]-based EGOFET. The red trace represents our free-coating reference device. All the measurements are recorded in MilliQ $\mathrm{H}_{2} \mathrm{O}$. 
(a)

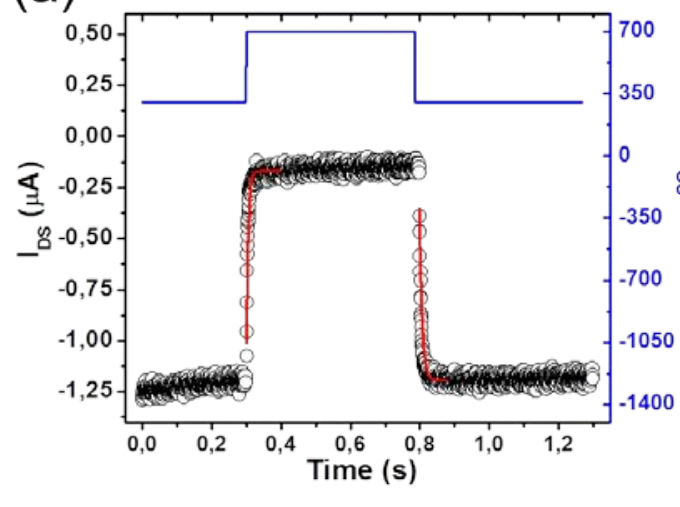

(b)

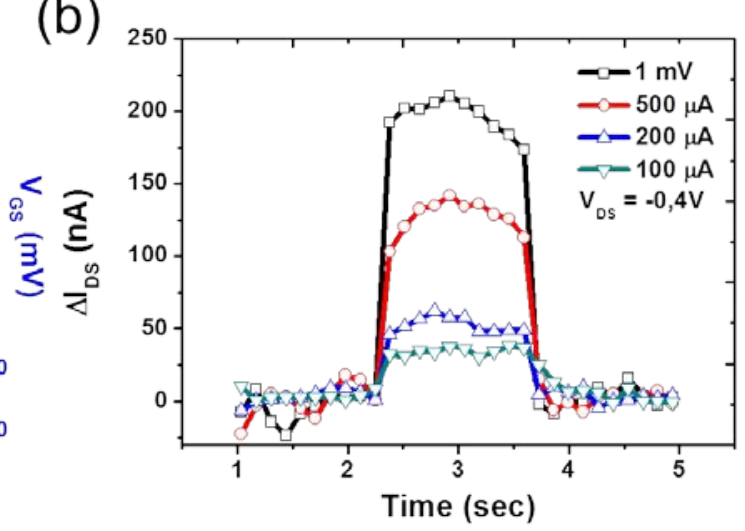

Figure 3. (a) Overlay of the output signal (black empty circles) according to the square pulse applied (blue line). The red lines stand for the exponential fits for charging/discharging processes (b) Overlay of current outputs due to $V_{G S}$ square pulses of amplitudes equal to $1 \mathrm{mV}$ (black), $500 \mu \mathrm{V}$ (red), $200 \mu \mathrm{V}$ (blue) and $100 \mu \mathrm{V}$ (green). All the measurements are recorded in MilliQ $\mathrm{H}_{2} \mathrm{O}$ at $V_{D S}=-0.4 \mathrm{~V}$. 

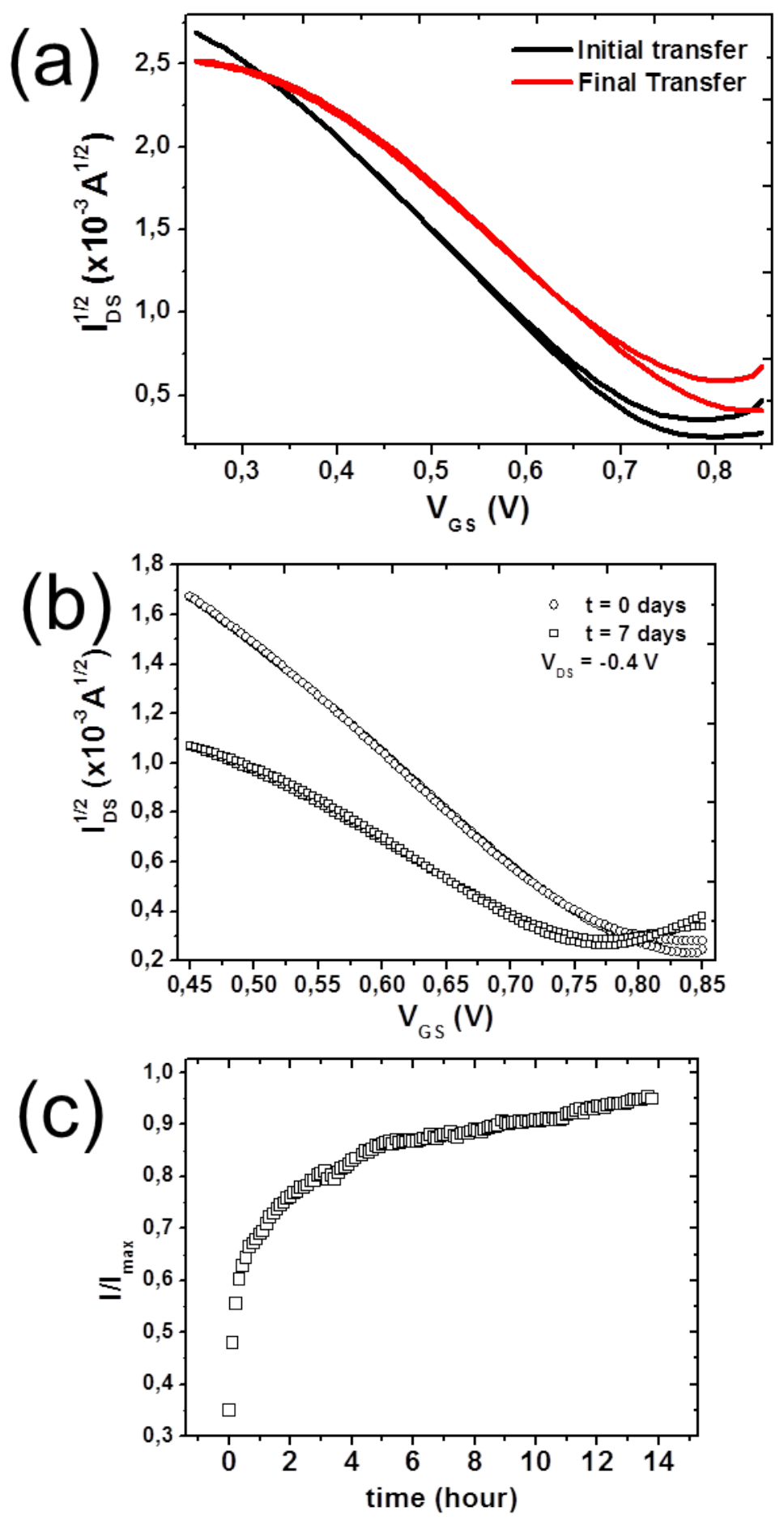

Figure 4. (a) Initial (black, $t=0 \mathrm{~min}$ ) and final (red, $t=60 \mathrm{~min}$ ) transfer characteristic of bias stress experiment $\left(V_{D S}=-0.4 \mathrm{~V}\right)$. (b) Shelf-stability of EGOFET device recorded at first and seventh day. (c) I-t plot of a real time monitoring $\left(V_{D S}=-0.4 \mathrm{~V} ; V_{G S}=0.35 \mathrm{~V}\right)$. All the measurements are recorded in MilliQ $\mathrm{H}_{2} \mathrm{O}$. 
Table 1. Mobility $(\mu)$, threshold voltage $\left(V_{t h}\right)$, transconductance $\left(g_{m}\right)$ and charge carrier density $\left(Q^{\prime}\right)$ for [DB-TTF:PS]-EGOFET.

\begin{tabular}{llll}
$\mu\left[\mathrm{cm}^{2} V^{-1} \mathrm{~s}^{-1}\right]^{\mathrm{a}}$ & $\mathrm{V}_{\mathrm{th}}\left[\mathrm{V}^{\mathrm{a}}\right.$ & $\mathrm{g}_{\mathrm{m}}[\mu \mathrm{S}]$ & $\mathrm{Q}^{\prime}\left[\times 10^{10} \mathrm{charge}_{\mathrm{cm}}{ }^{2}\right]$ \\
\hline $0,06( \pm 0,05)$ & $0,86( \pm 0,08)$ & 180 & 1,3 \\
\hline
\end{tabular}

[a] (listed values are averaged over 20 devices) 
This communication presents a novel Electrolyte Gated Field-Effect Transistor (EGOFET) based on a blend of dibenzo-tetrathiafulvalene and polystyrene deposited through Bar Assisted Meniscus Shearing (BAMS).This technique does not only capture industrial interest due to its scalability, but it allows the fabrication of high performing electronic devices suitable for (bio)-sensing applications. The reported devices can operate in aqueous solution with comparable complexity to real samples.

Keywords: Solution Shearing, Electrolyte-Gated Organic Transistors, Printable Electronics, Organic Semiconductor Blend, Organic Large-Area Electronics.

By Francesca Leonardi, Stefano Casalini, Qiaoming Zhang, Sergi Galindo, Diego Gutiérrez and Marta Mas-Torrent*

Electrolyte-Gated Organic Transistor based-on Solution Sheared Organic Semiconductor Blend

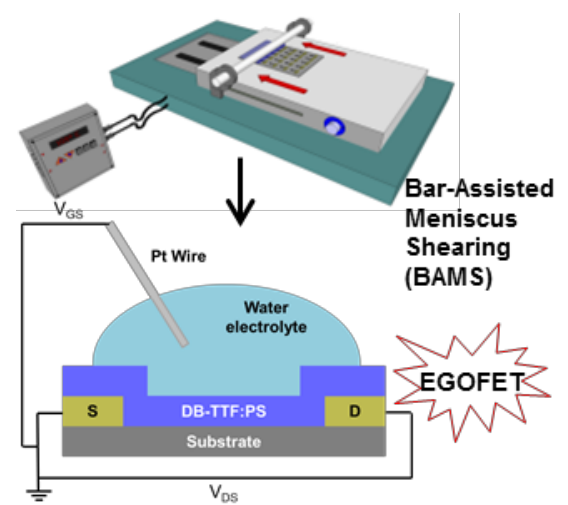


Supporting Information should be included here (for submission only; for publication, please provide Supporting Information as a separate PDF file).
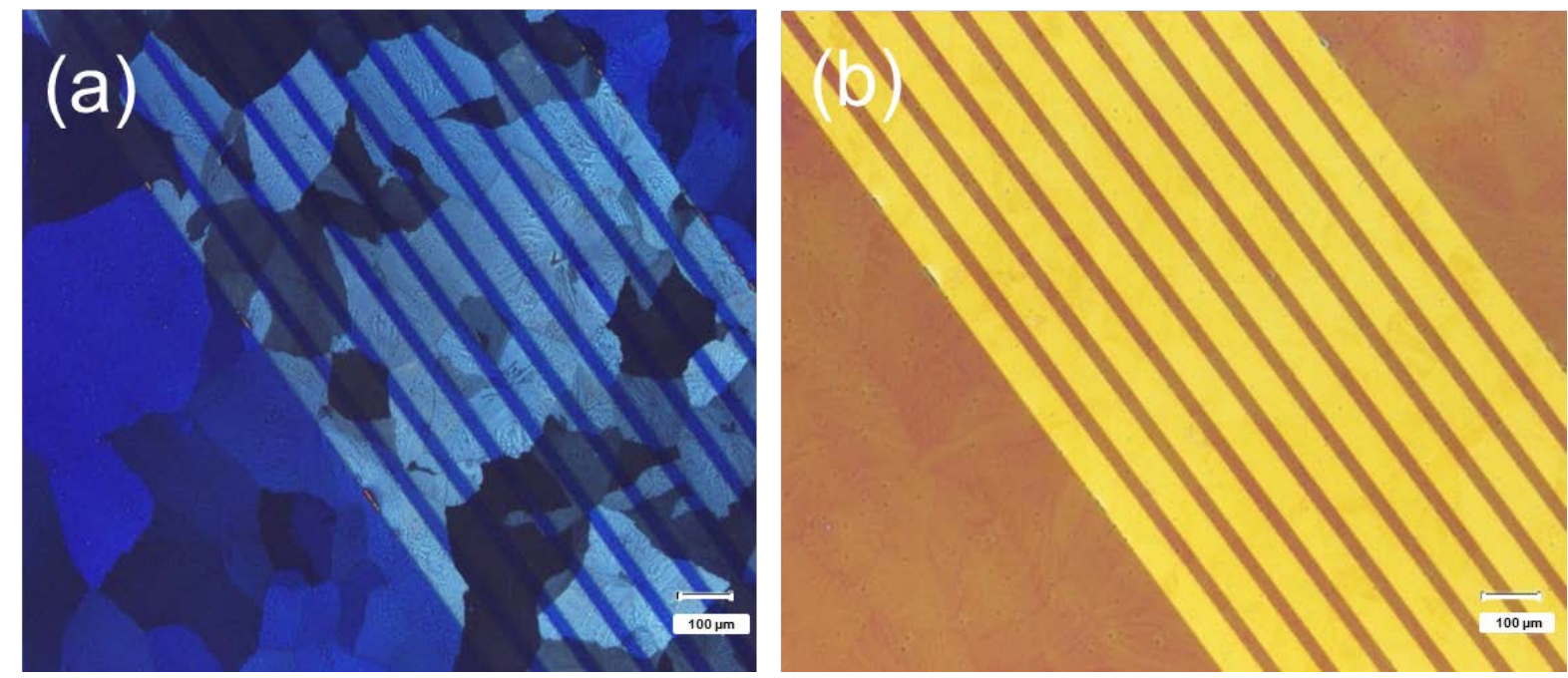

Figure S1. a) Optical polarized microscopy image of the OS blend thin-film acquired with crossed polarizer/analyzer setup. b) Corresponding optical image.
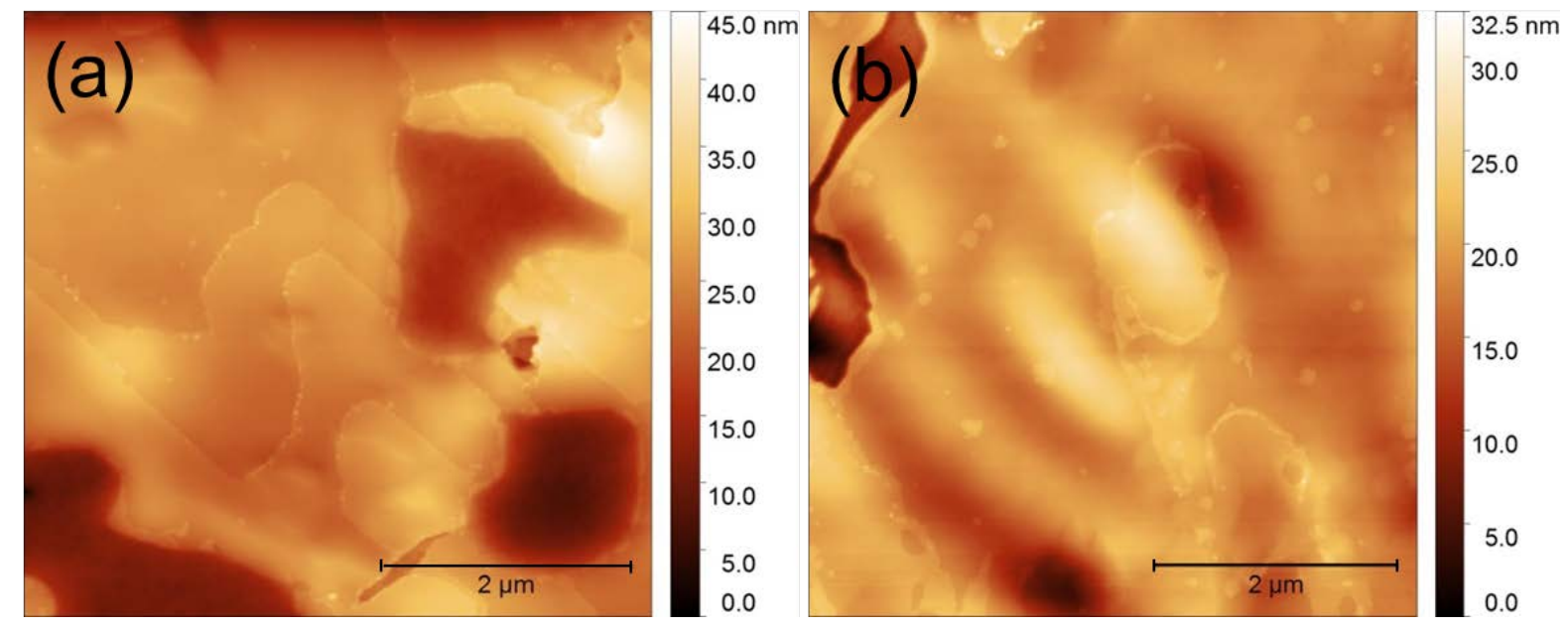

Figure S2. AFM images $\left(5 \times 5 \mu^{2}\right)$ of the DB-TTF:PS blend on the channel (a) and electrode (b) regions. The root mean square roughnesses are $8.1 \mathrm{~nm}$ and $5.6 \mathrm{~nm}$ respectively. 
(a)

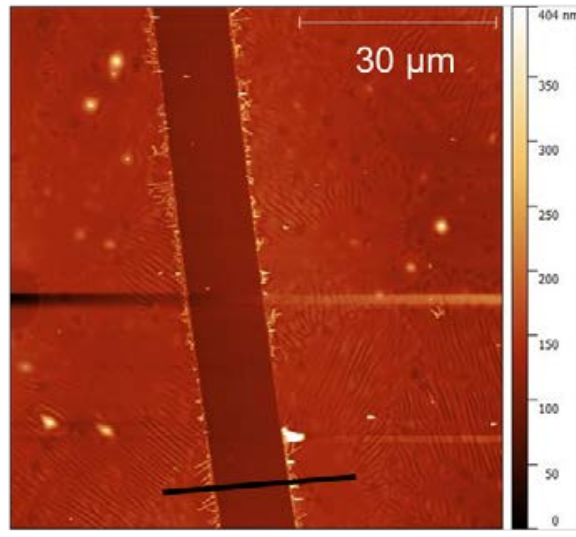

(b)

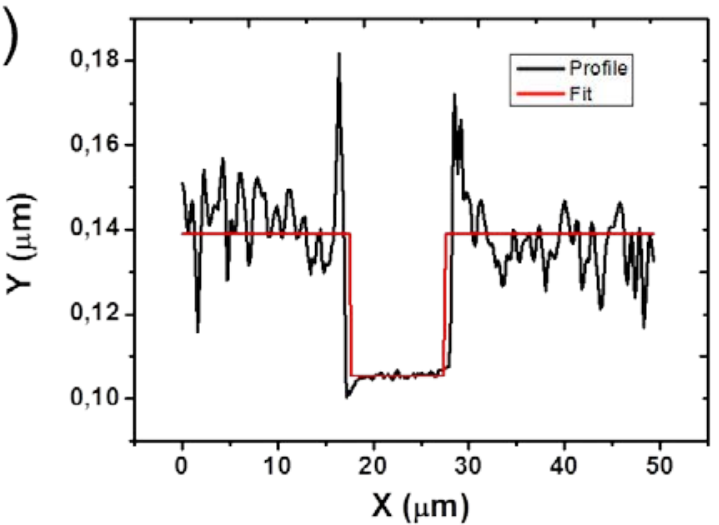

Figure S3 (a) AFM image of the scratch onto the DB-TTF:PS coating. (b) Profile plot related to the black line on the left image.

(a)

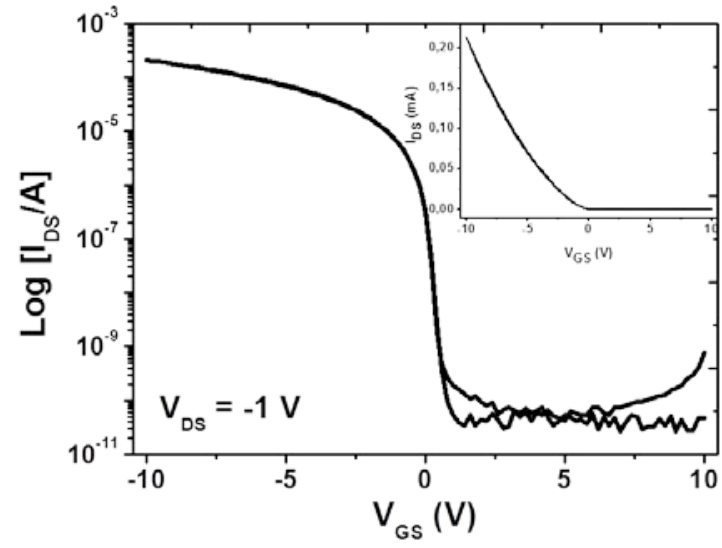

(b)

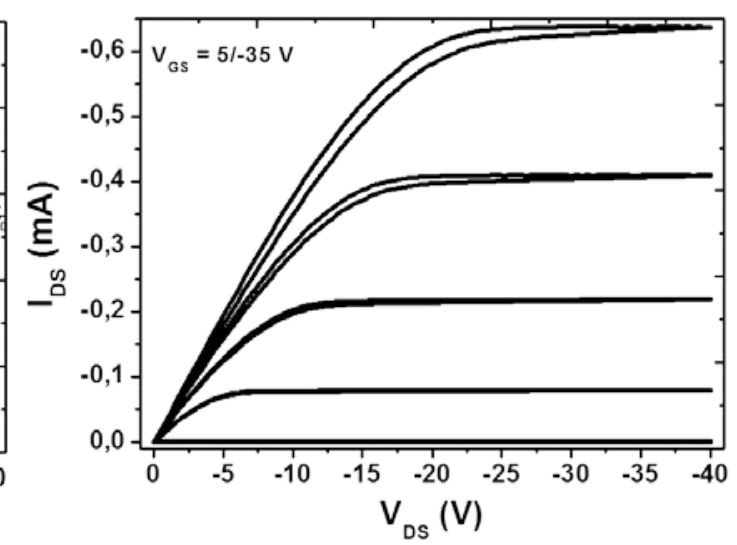

Figure S4. (a) Log-Lin plot of the transfer characteristics in bottom-gate bottom-contact configuration. The inset is the corresponding Lin-Lin plot. (b) I-V output characteristic with $V_{G S}$ spanning from +5 to $-35 \mathrm{~V}$ with pace of $10 \mathrm{~V}$.

(a)

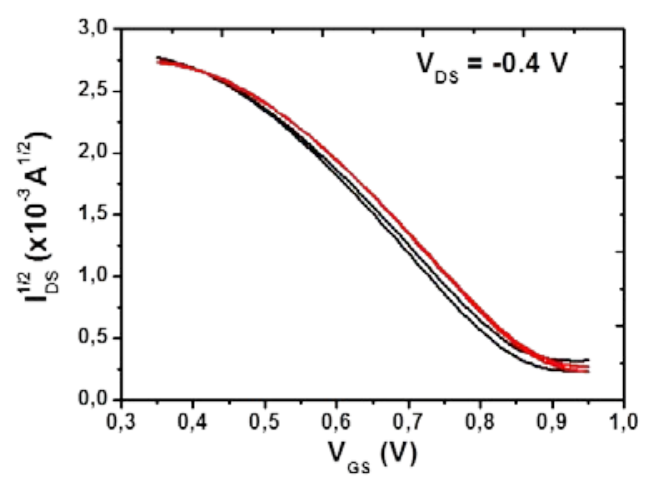

(b)

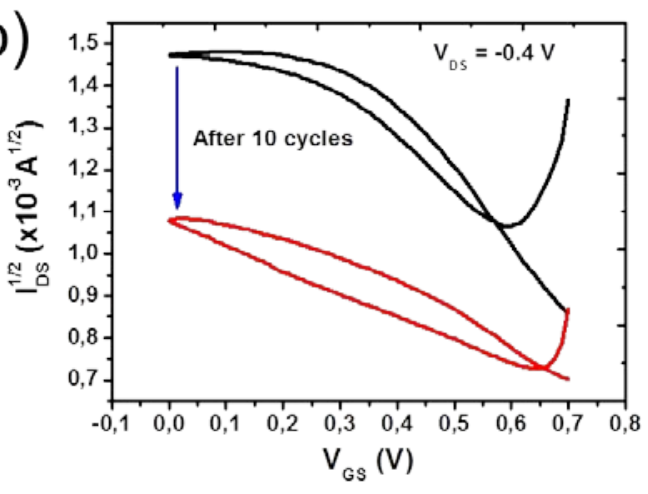

Figure S5. Comparison of I-V transfer characteristics of the (a)[DB-TTF:PS]-based EGOFET and (b) an EGOFET based on a PS-free coating of DB-TTF; after few cycles the device lose ist electrical modulation. 


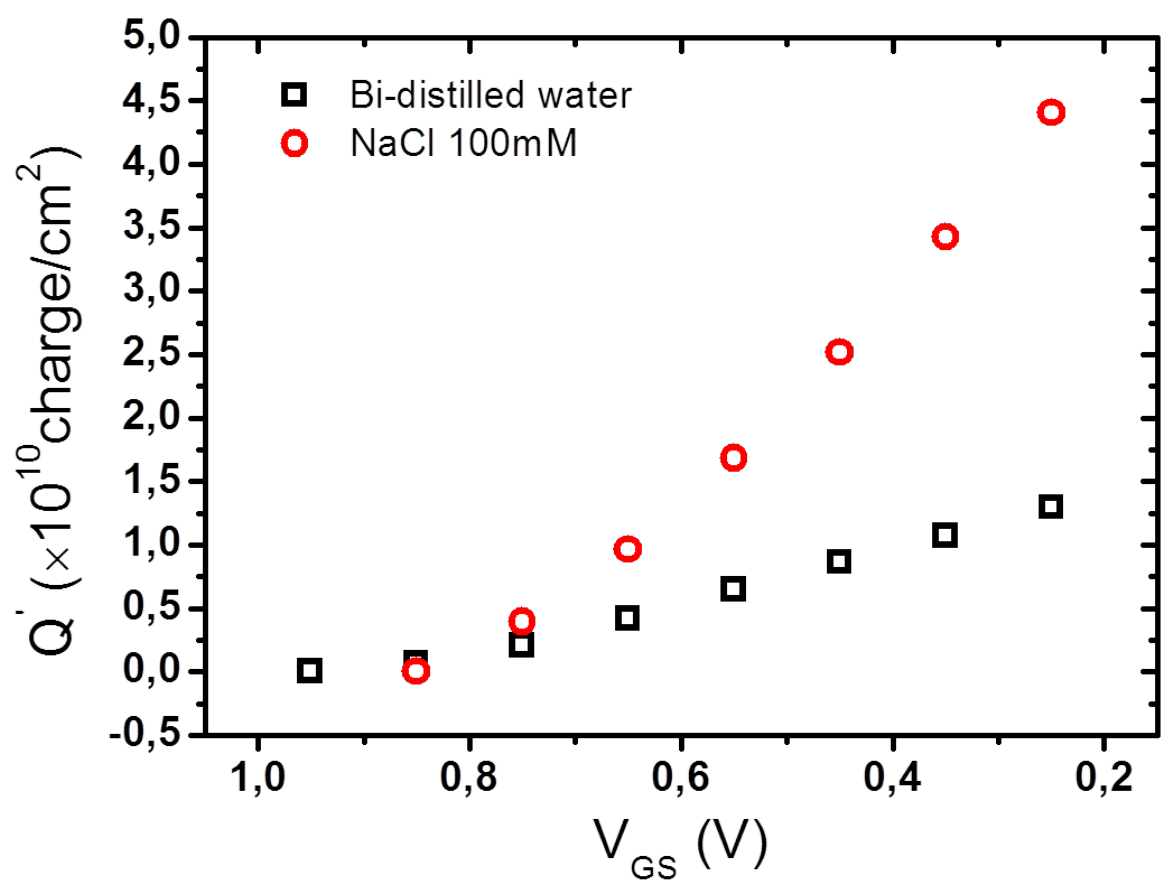

Figure S6. Plot of charge carriers density as a function of $V_{G S}$. 

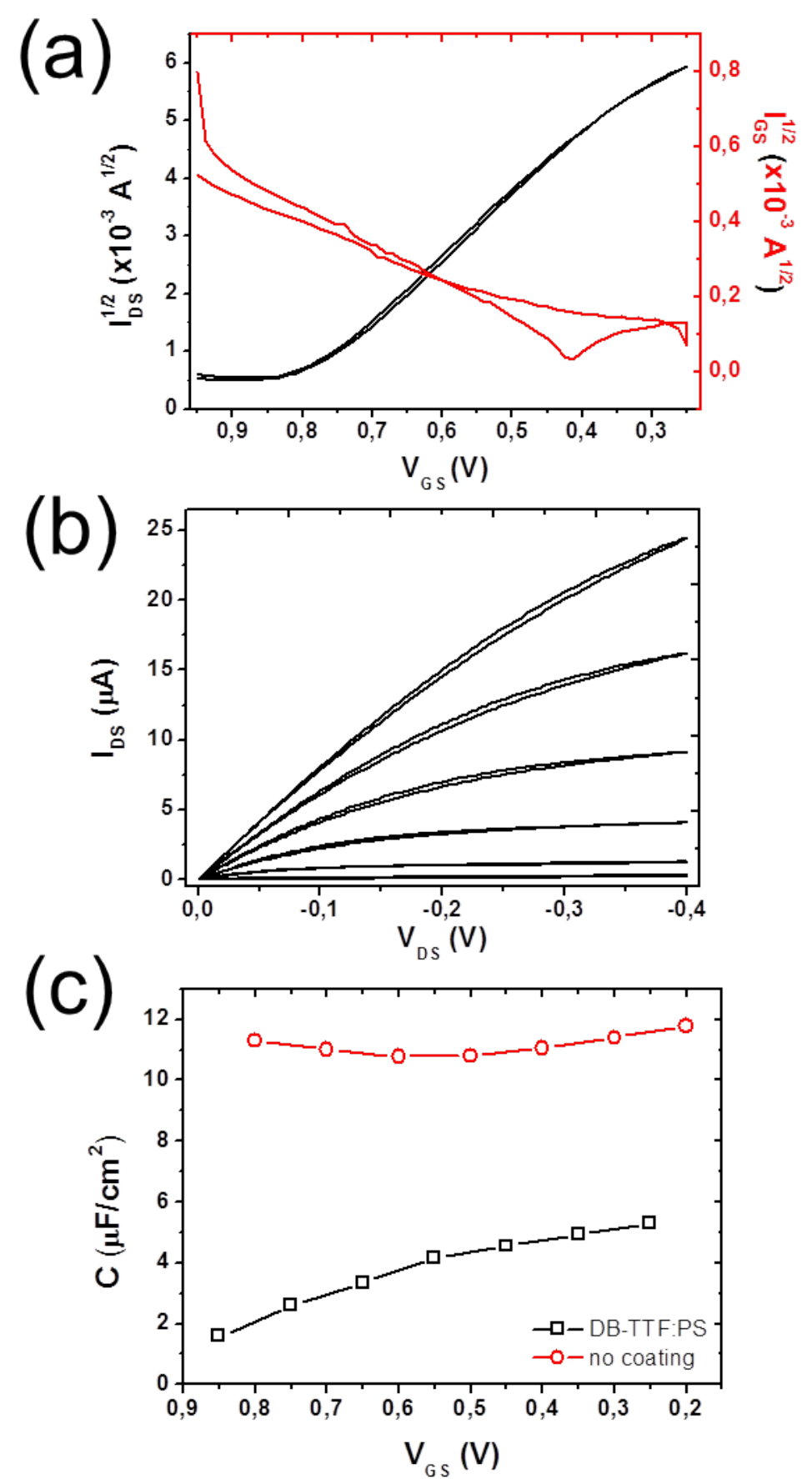

Figure S7. (a) I-V transfer characteristic of DB-TTF:PS EGOFET $\left(V_{D S}=-0.4 \mathrm{~V}\right)$; (b) I-V output characteristic $\left(V_{G S}=0.9 \mathrm{~V} \div 0.3 \mathrm{~V}\right.$ with step of $\left.0.1 \mathrm{~V}\right)$; (c) capacitance versus $V_{G S}$ plot of [DB-TTF:PS]-based EGOFET. The red trace represents our free-coating reference device. All the measurements are recorded in $\mathrm{NaCl} 100 \mathrm{mM}$. 


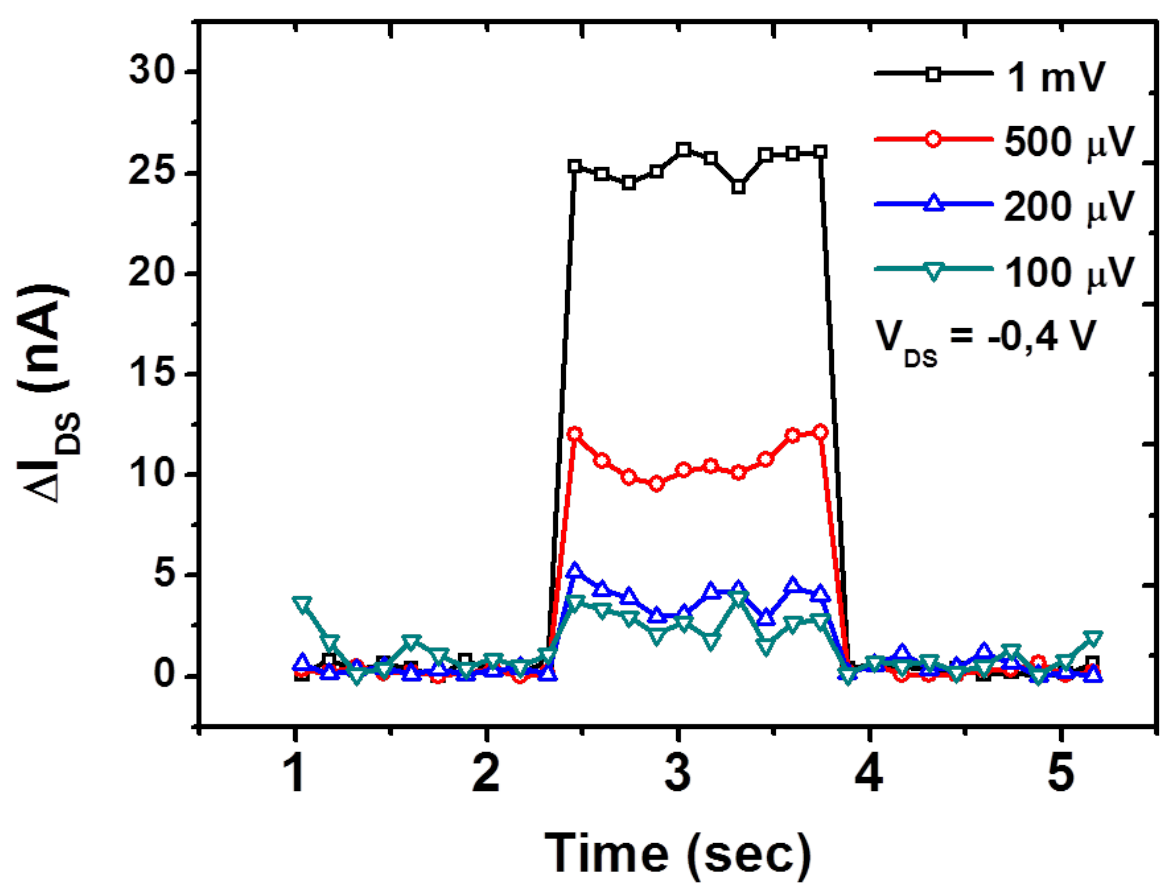

Figure S8. Overlay of current outputs due to $V_{G S}$ square pulses of amplitudes equal to $1 \mathrm{mV}$ (black), $500 \mu \mathrm{V}$ (red), $200 \mu \mathrm{V}$ (blue) and $100 \mu \mathrm{V}$ (green). Measurements are recorded in $\mathrm{NaCl}$ $100 \mathrm{mM}$. 

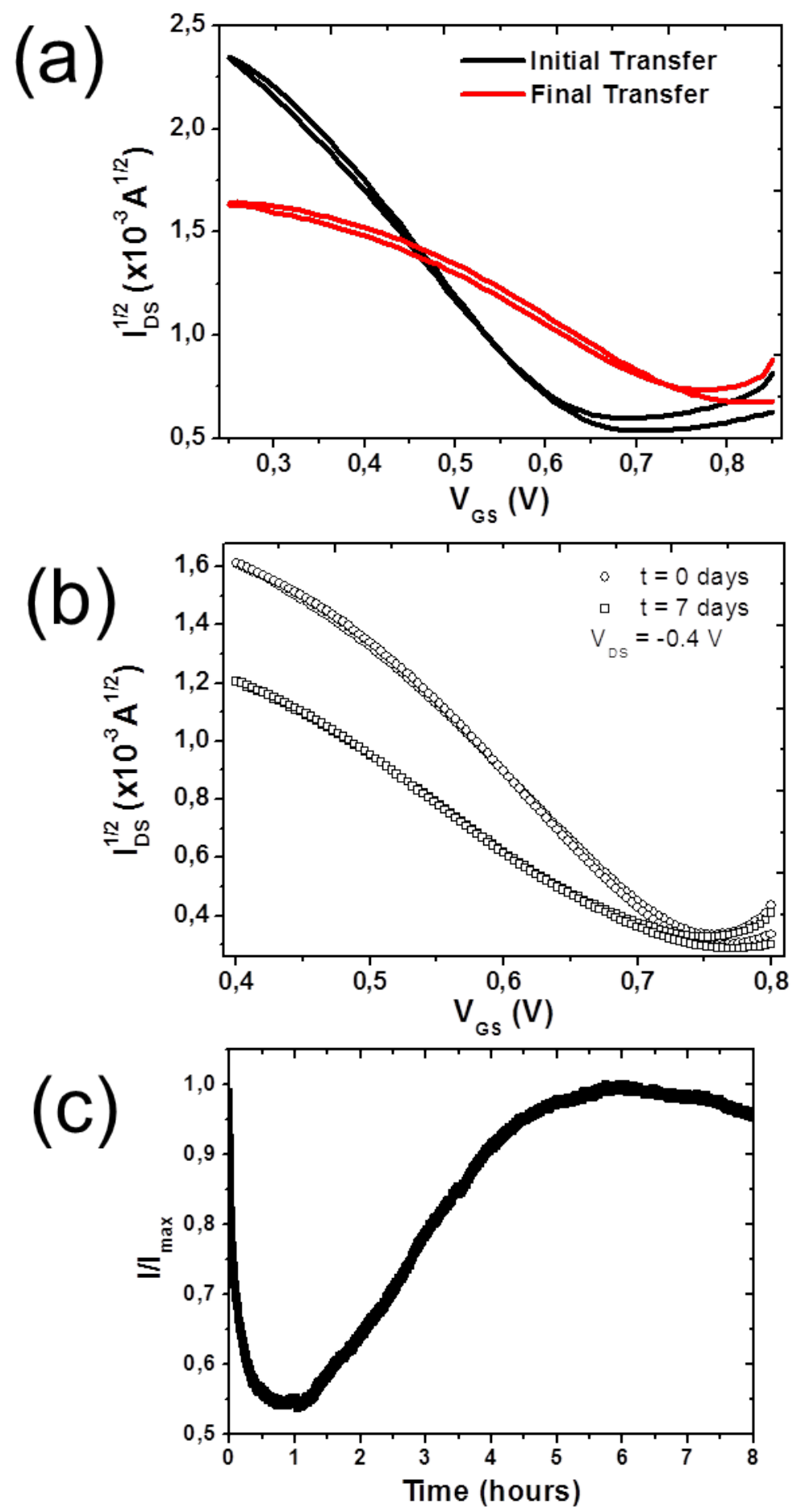

Figure S9. (a) Initial (black, $t=0 \mathrm{~min}$ ) and final (red, $t=60 \mathrm{~min}$ ) transfer characteristic of bias stress experiment $\left(V_{D S}=-0.4 \mathrm{~V}\right)$. (b) Shelf-stability of EGOFET device recorded at first and seventh day. (c) I-t plot of a real time monitoring $\left(V_{D S}=-0.4 \mathrm{~V} ; V_{G S}=0.35 \mathrm{~V}\right)$. All the measurements are recorded in $\mathrm{NaCl} 1 \mathrm{M}$. 